\title{
Machine Efficient Adaptive Image Matching Based on the Nonparametric Transformations
}

\author{
Bogusław Cyganek \\ AGH - University of Science and Technology \\ Al. Mickiewicza 30, 30-059 Kraków, Poland
}

\begin{abstract}
The paper presents machine efficient area-based image matching method that is based on a concept of matching-regions that are adaptively adjusted to image contents. They are in a form of square windows which grow to convey enough information for reliable matching. This process is controlled by local image contents. The images, however, are transformed into nonparametric representation. Such a liaison of information-theoretic models with nonparametric statistics allows for compensation for noise and illumination differences in the compared images, as well as for better discrimination of compared regions. This leads to more reliable matching in effect. Machine efficient implementation is also discussed in the paper. Finally the experimental results and conclusions are presented.
\end{abstract}

\section{Introduction}

The area-based image matching algorithms are ubiquities in image processing systems. They all operate in the similar fashion: the image regions of the same shape and size are compared by means of some measure. Then based on a result of this comparison the regions are pronounced to be matched or not. There are also many improvements to this basic scheme, since in practice the method is not always reliable. One of the most cumbersome problems is choice of the matching regions beforehand. Their shape and size determine quality of matching and unfortunately depend greatly on image contents, as well as on noise, texture, and image distortions. One of such improvements consists of adaptively adjusted matching regions to accommodate diverse image contents. There are different strategies that control this process. One of the very original solutions with a statistical model was proposed by Kanade and Okutomi [8]. The appropriate window is selected by evaluating the variations in intensity and disparity. Their method is based on the observation that at discontinuities the intensity and disparity variations are larger, unlike at the positions of surfaces. However, the implementation of this method is quite complicated.

The adaptive window technique developed by Lotti [9] for matching aerial images bases on window adjusting that is limited by edges and statistical contents of the matching regions. However, this technique is quite time consuming and rather limited to special class of images.

There are also many other techniques [3], such as multiple windowing [6] or a variable window concept for integral images proposed by Veksler [10]. 
In this paper we present an extension to the adaptive window growing technique (AWG) originally proposed in [3]. This method tries to adjust size of the matching window in order to conveyed enough information for more reliable matching. At the same time size of a window is kept as small as possible. However, in respect to [3], the technique has been simplified for easier implementation and speed up.

It can be shown that the AWG technique is equivalent to computation of signal entropy in a window, however in a more efficient way. This is achieved thanks to the prior image transformation into nonparametric representation which is more resistive to noise and local lighting incompatibilities. Such domain allows for fast information assessment since this nonparametric signal representations conveys mutual relation among neighboring pixels.

\section{The Adaptive Window Concept for Image Matching}

Zabih and Woodfill proposed the nonparametric Rank and Census transforms for computation of correspondences. The Census, used in the presented method, returns an ordered stream of bits, defined as follows: a bit at a given position is ' 1 ' if and only if an intensity value at a pixel is greater or equal to the central pixel; otherwise a bit is set to ' 0 '. Local neighborhood of pixels is usually $3 \times 3$ and $5 \times 5$ square window [11].

The consecutive image matching, in the nonparametric domain, is usually done by means of Hamming measure used to compare different image areas [11][1], although other measures are possible as well [5]. The AWG technique presented in this paper allows for better control of the size of matching windows (whereas size of the Census transform is fixed to $3 \times 3$ neighborhoods and should not be confused hereafter).

For a given central pixel at $(i, j)$ and surrounding $n \times n$ square neighbourhood, the corresponding Census measure $I C(i, j)$ can be defined as a series of bits [3][5]:

$$
\operatorname{IC}(i, j)=b_{n^{2}-1} \ldots b_{k} \ldots b_{3} b_{2} b_{1} b_{0} \text {, where } k \in\left[0, \ldots, n^{2}-1\right] /\left\{\left\lfloor\frac{n^{2}}{2}\right\rfloor\right\} .
$$

The $b_{k}$ parameter can be expressed as follows:

$$
b_{k}=1 \quad \text { if } \quad I\left(i-\left\lfloor\frac{n}{2}\right\rfloor+\left\lfloor\frac{k}{n}\right\rfloor, j-\left\lfloor\frac{n}{2}\right\rfloor+k \bmod n\right) \geq I(i, j), 0 \text { otherwise, }
$$

where $I(i, j)$ denotes the intensity value for an image at a point at $(i, j)$ in image coordinates, $\lfloor k / n\rfloor$ integer division of $k$ by $n, \mathrm{k} \bmod \mathrm{n}$ modulo $n$ division.

Let us examine some $3 \times 3$ pixel neighbourhoods and their Census representations (Fig. 1). Not knowing values of pixels surrounding this neighbourhood, the complete Census value can be estimated only for the central pixel from the neighbourhood of interest. Therefore all the other pixels have their Census values assessed only in respect to their closest neighbouring pixels belonging to this $3 \times 3$ neighbourhood. All other values are left undefined and marked as ' $x$ ' (don't care). Such bits can be assigned ' 0 ' or ' 1 ' depending on intensity values of other pixels, lying in other $3 \times 3$ neighbourhoods, where the given pixel in turn lies in their centre. The function $q\left({ }^{\prime} b\right.$ ') in Fig. 1 returns number of bits with value 'b' (i.e. number of ' 0 s' or '1s'). 
The question arises on distribution of the Census values, computed in local $3 \times 3$ neighborhoods for the whole image, in respect to the distribution of intensities in the input image. It is well known that the latter greatly influences any subsequent matching, no matter what matching criteria are taken into consideration. The answer to this question is fundamental to the AWG technique described in this paper.

Based on definition (1) we observe that neighborhoods with constant intensity produce bit streams with all bits set to ' 1 ' (Fig. 1a). That is data has zero entropy and the conveyed information is too unreliable for matching. However, in Fig. 1b and Fig. 1c we notice that with an increase of spatial distribution - counted as an entropy of intensity values $-q\left({ }^{\prime} 0\right.$ ') increases as well. For exemplary values in Fig. 1 the entropy of data in selected $3 \times 3$ regions increases conveying more information that in consequence allows for more reliable matching.

For any $n \times n$ neighborhood it is evident that the following relation holds:

$$
q\left({ }^{\prime} 0^{\prime}\right)+q\left({ }^{\prime} 1^{\prime}\right)+q\left({ }^{\prime} x^{\prime}\right)=B n^{2},
$$

where $B$ stands for number of bits assigned for Census representation of a pixel. Therefore maximization of $q\left({ }^{\prime} O\right.$ ') can be achieved by keeping $q($ ' 1 ') at minimum and when undefined bits ' $x$ ' become ' 0 '. This feature is used in implementation.

Pixel neighborhoods in the input image (intensity values set for example)

a)

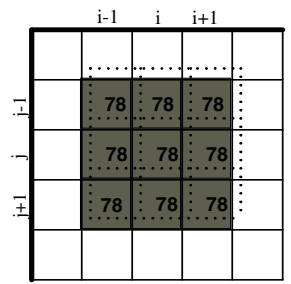

b)

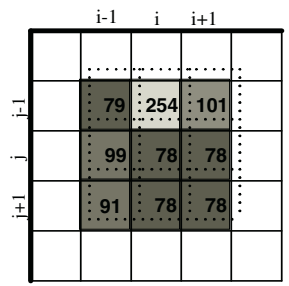

c)

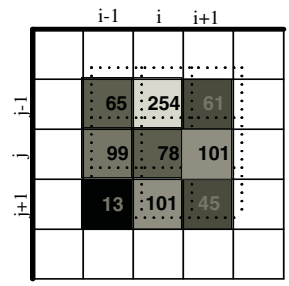

Pixel neighborhoods after $3 \times 3$ Census transformation
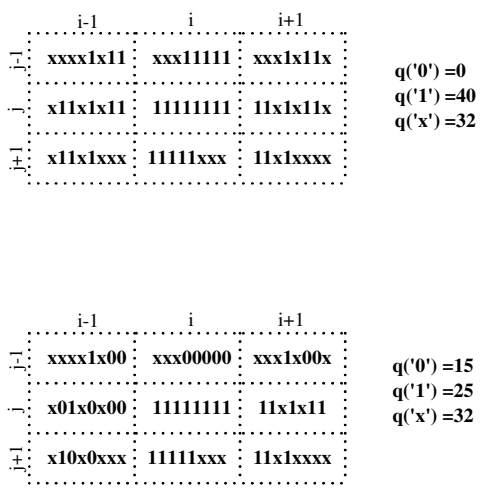

$\mathbf{q}\left(\mathbf{0}^{\prime}\right)=15$

$\mathbf{q}\left({ }^{\prime} 1{ }^{\prime}\right)=25$ $\mathbf{q}\left(' x^{\prime}\right)=32$

$\mathbf{q}\left(\mathbf{\prime}^{\prime}\right)=\mathbf{1 8}$

$\mathbf{q}\left({ }^{\prime} 1{ }^{\prime}\right)=22$

$\mathbf{q}\left(\mathbf{x}^{\prime}\right) \mathbf{= 3 2}$

Fig. 1. Some $3 \times 3$ neighborhoods and their Census representations. $q$ (' $b$ ') returns number of bits with value ' $b$ '. Intensity values set only as an example - relevant are only their mutual relations 
Disambiguation of all Census values in any $3 \times 3$ neighborhood requires knowledge of intensity values of all pixels surrounding this neighborhood. Such a situation with some exemplary intensity values is presented in Fig. 2 . We notice that an increase of the $q($ ' $O$ ') entails a further increase of the entropy of data in the pixel neighbourhoods of interest. For $3 \times 3$ neighborhoods around a point at indexes $(i, j)$ in Fig. 2, the entropies are respectively (from the top): $0.29,0.41$, and 0.55 .

Pixel neighborhoods in the input image (intensity values set for example)

a)

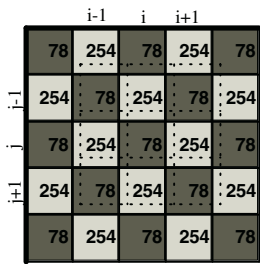

b)

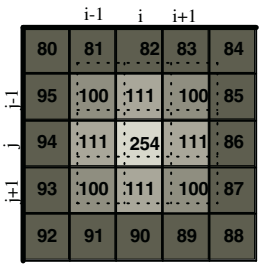

c)

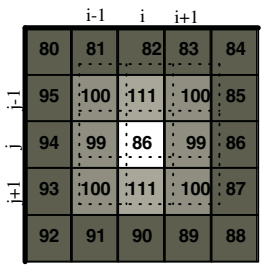

Pixel neighborhoods after $3 \times 3$ Census transformation
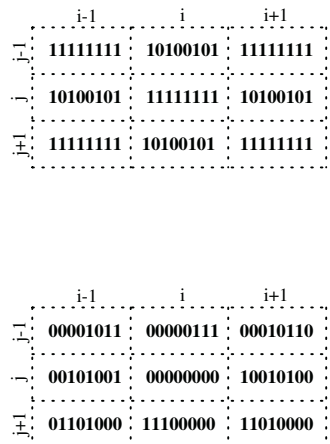

$q\left({ }^{\prime} 0^{\prime}\right)=48$ $q\left({ }^{\prime} 1 '\right)=24$

$\mathbf{q}\left({ }^{\prime} \mathbf{0}^{\prime}\right)=\mathbf{5 2}$ $\mathbf{q}\left({ }^{\prime} 1^{\prime}\right)=20$

Fig. 2. The specific $3 \times 3$ pixel neighborhoods and their Census transformations. Relevant are relations of intensity values (set for example here)

From the definition (1) and from the mutual relation between the central point ' $\mathrm{X}$ ' and all its closest neighbors ' $a$ ' - ' $h$ ' in Fig. 3 we see that if only an intensity value of the point ' $\mathrm{X}$ ' is different from all values of the points ' $a$ ' - ' $h$ ' we have met the condition for maximum entropy for this point arrangement, and at the same time the maximum number of ' 0 's is achieved. There exists yet an another type of the mutual pixel relation - the relation among three consecutive pixels, such as ' $a$ ', ' $b$ ', and 'd' in Fig. 3. For each pair of neighboring pixels from such a triple, a bit with value ' 0 ' can be assigned if and only if any pair of intensity values is different. In such a case, for each pair of pixels from such a triple, one Census representation obtains ' 1 ' at the corresponding bit position, while the same bit position - but in the complementary pixel from this pair - holds ' 0 '. This is clear from (2) since there is an exclusive 
relation. Thus, if only the abutted pixels have different values, at the pertaining bit position the only possibility is: bit ' 1 ' in the first Census pixel of a pair and ' 0 ' for the second one.

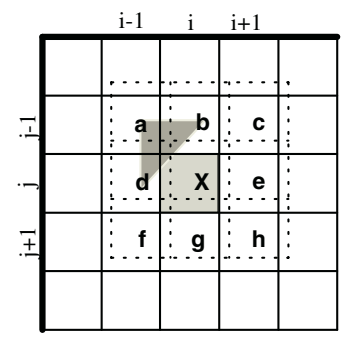

Fig. 3. Relations among pixel values in the Census transformation

The rules for the maximum number of ' 0 s' - and in consequence entropy increase - in a Census representation of any $3 \times 3$ neighborhood (see Fig. 3 ) are as follows:

1. A value of the central pixel $\mathbf{X}$ is irrelevant provided that it is different than any other pixel from its closest neighborhood (i.e. from the all pixels $\mathbf{a}, \mathbf{b}, \mathbf{c}, \mathbf{d}, \mathbf{e}, \mathbf{f}, \mathbf{g}$, and $\mathbf{h}$ in Fig. 3):

$$
\forall p \in N-\{X\}: I(p) \neq I(X)
$$

where $I(p)$ is an intensity value of a pixel at index $p$ (see Fig. 1b) from the $3 \times 3$ neighborhood $N$.

2. Pixel values of any corner-triple (such as e.g. a, b, d in Fig. 3) must be different, i.e.:

$$
I(a) \neq I(b) \neq I(c) .
$$

3. All other pixels bordering with pixels from the neighborhood $N$ must have their intensity values less than their direct pixels-neighbors from $N$. For example, for the pixel $\mathbf{b}$ in Fig. 3 these would be pixels at indexes: (j-2,i-1), (j-2,i), and $(j-2, i+1)$.

The upper bound for any $3 \times 3$ Census neighbourhood $q_{\max }\left({ }^{\prime} O\right.$ ') $=52$ can be easily found from the conditions 1-3 and after observing Fig. 3. This with (3) corresponds with lower bound $q_{\min }(' 1$ ') $=20$. The lower bound for data entropy (LBE) in any $3 \times 3$ Census neighbourhood that preserves the conditions 1-3 is obtained by taking only four different values and can be easily found from (4) to be: $\mathrm{LBE}=-4 / 9 * \log 4 / 9$ $2 * 2 / 9 * \log 2 / 9-1 / 9 * \log 1 / 9 \approx 0.55$. The upper bound for the entropy in any $3 \times 3$ neighbourhood is $\mathrm{MBE}=\log (9) \approx 0.95$ (i.e. nine different values). Thus, any $3 \times 3$ neighbourhood of pixels that preserve conditions 1-3 guarantee almost $58 \%$ of maximum possible entropy in this neighbourhood. Concluding we can state that the set of conditions 1-3 guarantying the maximum number of ' 0 ' $s$ (or equivalently the minimal number of ' 1 's) in any $3 \times 3$ Census neighborhood leads to at least LBE. Therefore the sought size $n$ of a matching square window can be found as to 
maximize the averaged (per pixel) value of $q_{a v}(' 0$ ') in a window of interest with a constraint $q_{\text {tot }}\left({ }^{\prime} \mathrm{O}^{\prime}\right)>\vartheta_{0}=0$ :

$$
n: \max _{n_{\min } \leq n \leq n_{\max }} q_{a v}\left(\mathrm{O}^{\prime}\right) \wedge q_{t o t}\left(\mathrm{O}^{\prime}\right)>\vartheta_{0}=0
$$

where $n_{\min }$ and $n_{\max }$ set the lower and upper bound of size of the matching window, $\theta_{0}$ is a certain threshold for a total minimal amount of ' 0 s' in a window of interest; $\theta_{0}$ usually is set to 0 . Thus, applying (6) we obtain the minimal size of the matching window that is adapted to convey as much as possible of information to allow a more reliable matching. Otherwise, in a case of a constant intensity we obtain information that there is no such a window that can guarantee a reliable matching.

The other possibility is to allow window growing up to such a minimal size $n$ for which the achieved average $q_{\text {tot }}\left(' 0\right.$ ') reaches a predefined threshold value $\vartheta_{1}$ :

$$
\min (n): q_{a v}\left({ }^{\prime} 0^{\prime}\right)>\vartheta_{1}
$$

Similarly, one can search for such a minimal size $n$ for which the total $q_{\text {tot }}$ (' 0 ') reaches a predefined threshold value $\vartheta_{2}$ as follows:

$$
\min (n): q_{t o t}\left(0^{\prime}\right)>\vartheta_{2}
$$

Computations of (7) and (8) are simpler and in consequence faster than (6). However they require an arbitrary setting of the threshold values.

\section{Experimental Results}

Experimental results and discussion of the image matching based on AWG operating in accordance with (6) can be found in [3]. In this section we present results that help compare different versions of the AWG given by (6)-(8). The computational platform consists of the IBM PC with Pentium 4 with $3.4 \mathrm{GHz}, 2 \mathrm{~GB}$, implementation in $\mathrm{C}++$,.

Fig. 4 presents results of the AWG technique applied to match the "PCB" stereo pair of size $253 \times 193$ (a). The disparity map obtained in accordance with (6) is

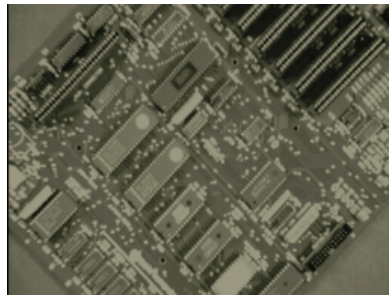

a

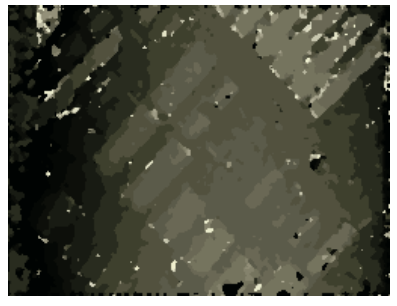

b

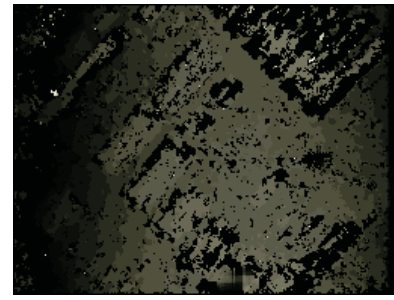

c

Fig. 4. The AWG technique applied to the "PCB" stereo image $(253 \times 193)$ : left image of a pair (a), disparity map from formula (6) (b), disparity map from formula (7) and $\theta_{1}=2$ (c) 
depicted in (b) while disparity from (7) with $\theta_{1}=2$ in (c). Execution times are 1.75 and $1.68 \mathrm{~s}$, respectively. Parameter $\theta_{1}$ was chosen based on prior experiments. Allowable window growing spanned: $n_{\min }=3$ to $n_{\max }=30$.

Fig. 5 contains results of the AWG applied to the "Mask" $450 \times 375$ pair (a). Disparity map from (6) is shown in (b) and with formula (7) with $\theta_{1}=2$ in (d), formula (8) with $\theta_{2}=15$ in (e), and $\theta_{2}=150$ in (f). Execution times are as follows: 2.05 for (b), 1.99 for (d), 0.83 for (e), and 4.22 for (f). The latter caused by the fact of much higher matching windows due to high $\theta_{2}$. However, execution of (e) compared to (d) is match faster, because total amount of ' $0 s^{\prime}$ ' is easier to compute than their average.

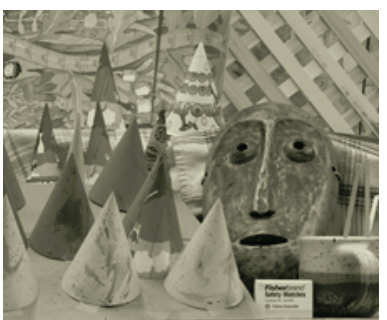

a

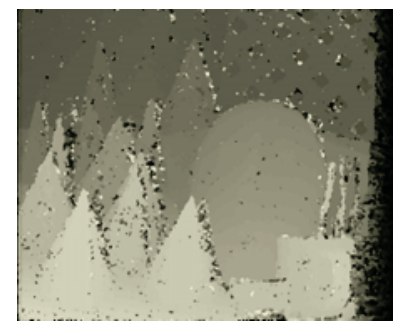

d

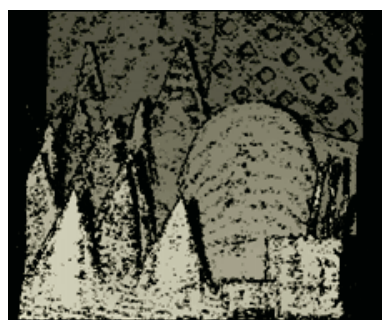

b

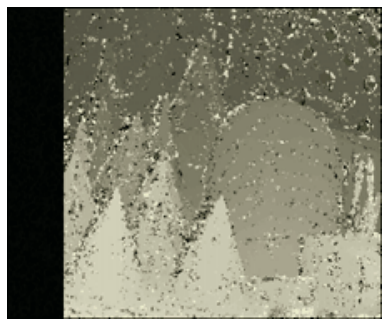

e

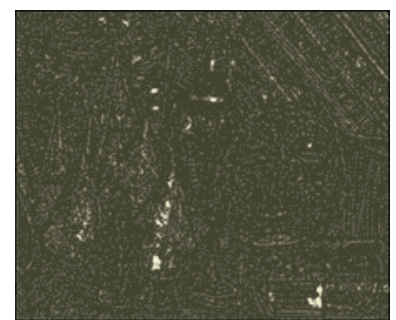

c

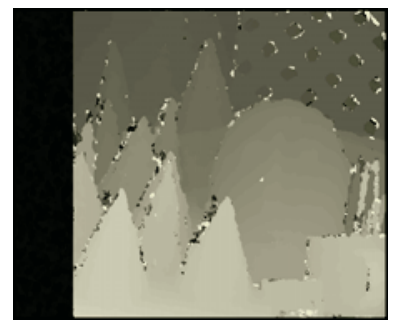

f

Fig. 5. The AWG technique applied to the "Mask" pair $(450 \times 375)$ : left image of a pair (a), disparity map (6) (b), visualization of adaptive windows (lither places denote bigger windows) (c), disparity map with formula (7) and $\theta_{1}=2$ (d), disparity map with formula (8) and $\theta_{2}=15$ (e), disparity map with formula (8) and $\theta_{2}=150$ (f)

For each pixel location the size of a matching window is determined with the AWG technique defined by (6). Disparity values are found for each pixel by matching square windows of sizes appropriately set in the previous step. As a matching measure the Hamming distance is computed from the Census representation of each pair of candidate windows. Matching is done with a fast block matching algorithm based on the winner-update strategy with hashing table [2]. The visible limited occlusions are due to a simple stereo algorithm without the cross-checking [12]. In Fig. 5(d-f) we notice also many occluding areas due to large displacement between images of this stereo pair. However, the details were preserved due to adaptively chosen matching windows.

The worst case time complexity of the AWG algorithm (6)-(8) can be estimated as follows: 


$$
O\left(N M n_{\max }^{2}\right)
$$

for image of size $N \times M$ pixels, where $n_{\max }$ is the maximum allowable matching window. However, the arithmetic operations are limited only to integer comparisons and summations. Therefore the technique fits well hardware implementations.

\section{Conclusions}

The paper presents the image matching technique with adaptively changed matching areas. The whole matching is performed after changing the original images into nonparametric domain by means of the Census transformation. The basics of the AWG technique were presented which can be summarized as follows: an increase of the zero-value-bits $q\left({ }^{\prime} O\right.$ ') in any Census neighborhoods indicates increase of entropy in this neighborhood of pixels. Different mutations (6)-(8) of the method allow for adjustment of the matching region at different computational cost. In effect, the subsequent matching is much more reliably than in a case of fixed windows [3].

The experimental results with the AWG technique applied to the stereo matching showed robustness of this technique. The main purpose of avoiding low-textured areas was achieved by means of very simple integer arithmetic. Therefore the AWG method seems to be appropriate for hardware and real-time implementations.

\section{Acknowledgement}

This paper has been sponsored by the Polish Committee of Scientific Research (KBN) grant number: 3T11C 04526.

\section{References}

1. Banks J., Bennamoun M., Corke P.: Non-Parametric Techniques for Fast and Robust Stereo Matching. CSIRO Manufacturing Science and Technology, Australia (1997)

2. Chen, Y-S., Hung, Y-P., Fuh, C-S.: Fast Block Matching Algorithm Based on the WinnerUpdate Strategy. IEEE Trans. On Image Processing, Vol. 10, No. 8, (2001) 1212-1222

3. Cyganek, B.: Adaptive Window Growing Technique for Efficient Image Matching. Technical Report, AGH-University of Science and Technology, Krakow, Poland (2004)

4. Cyganek, B., Borgosz, J.: Maximum Disparity Threshold Estimation for Stereo Imaging Systems via Variogram Analysis. ICCS 03, Russia/Australia (2003) 591-600

5. Cyganek, B.: Comparison of Nonparametric Transformations and Bit Vector Matching for Stereo Correlation. Springer LNCS 3322 (2004) pp. 534-547

6. Fusiello, A. et.al.: Efficient stereo with multiple windowing. CVPR 858-863 (1997)

7. Haykin, S.: Neural Networks. A Comprehensive Foundation. Prentice-Hall (1999)

8. Kanade, T., Okutomi, M.: A stereo matching algorithm with an adaptive window: Theory and experiment. PAMI, 16(9) (1994) 920-932

9. Lotti, J-L., Giraudon, G.: Adaptive Window Algorithm for Aerial Image Stereo. INRIA Technical Report No 2121 (1993) 
10. Veksler, O.: Fast Variable Window for Stereo Correspondence using Integral Images. Computer Vision and Pattern Recognition (2003)

11. Zabih, R., Woodfill, J.: Non-Parametric Local Transforms for Computing Visual Correspondence. Proc. Third European Conf. Computer Vision (1994) 150-158

12. Zhengping, J.: On the Mutli-Scale Iconic Representation for Low-Level Computer Vision. $\mathrm{Ph} . \mathrm{D}$. Thesis. The Turing Institute and University of Strathclyde (1988) 114-118 